\title{
Ein palliativer Ansatz für schwerste Anorexia nervosa?
}

Westermair, Anna Lisa ; Perrar, K M ; Schweiger, U

\begin{abstract}
Even with guideline-conform treatment in specialized outpatient clinics and intensive treatment programs, some patients suffering from severe and enduring anorexia nervosa do not achieve a satisfying level of mental health and quality of life. As this patient group is associated with a high mortality, a palliative stage of anorexia nervosa is postulated. In these cases, treatment decisions should factor in not only a prolongation of life but also quality of life, especially when compulsory treatment is considered. In doubtful cases, a palliative care physician and the clinical ethics committee should be consulted.
\end{abstract}

DOI: https://doi.org/10.1007/s00115-020-00875-3

Other titles: A palliative approach for severest anorexia nervosa?

Posted at the Zurich Open Repository and Archive, University of Zurich ZORA URL: https://doi.org/10.5167/uzh-192109

Journal Article

Accepted Version

Originally published at:

Westermair, Anna Lisa; Perrar, K M; Schweiger, U (2020). Ein palliativer Ansatz für schwerste Anorexia nervosa? Der Nervenarzt, 91(5):411-416.

DOI: https://doi.org/10.1007/s00115-020-00875-3 
Autoren: Anna Lisa Westermair ${ }^{1}$, Klaus Maria Perrar ${ }^{2}$, Ulrich Schweiger ${ }^{1}$

${ }^{1}$ Klinik für Psychosomatik und Psychotherapie, Universitäts-Klinikum Schleswig-Holstein (UKSH) Campus Lübeck

${ }^{2}$ Zentrum für Palliativmedizin, Uniklinik Köln

Korrespondierender Autor:

Dr. med. Anna Lisa Westermair

Ratzeburger Allee 160, 23538 Lübeck

045150098710

anna.westermair@uksh.de

Titel: Ein palliativer Ansatz für schwerste Anorexia nervosa?

Zusammenfassung

Auch unter Leitlinien-gerechter Therapie in Spezialambulanzen und Intensivprogrammen erreichen manche PatientInnen mit Anorexia Nervosa kein zufriedenstellendes Ausmaß an seelischer Gesundheit und Lebensqualität. Diese Patientengruppe hat zudem eine hohe Mortalität, weswegen wir die Existenz eines Palliativstadiums der Anorexia nervosa postulieren. In dieser Situation sollte bei therapeutischen Entscheidungen neben einer Lebensverlängerung auch die Lebensqualität bedacht werden, insbesondere wenn Maßnahmen unter Zwang erwogen werden. Im Zweifelsfall empfiehlt sich die Hinzuziehung eines Palliativmediziners und des klinischen Ethikkomittees.

Schlüsselwörter

Palliativmedizin, Anorexia Nervosa, severe and enduring eating disorder, Lebensende, medical Futility

Title: A Palliative Approach for the Most Severe Presentations of Anorexia Nervosa?

\section{Abstract}

Even with treatment according to guidelines, with specialized outpatient clinics and intensive therapy programs, some patients suffering from severe and enduring anorexia nervosa do not achieve a satisfying level of mental health and quality of life. As this is associated with high mortality, we postulate a palliative stage of anorexia nervosa. In these cases, therapeutic decisions should factor in not only prolongation, but also quality of life, especially when compulsory treatment is considered. In case of doubt, a palliative care professional and the clinical ethics committee should be consulted.

Keywords

Palliative care, Anorexia Nervosa, severe and enduring eating disorder, end of life, medical futility 
Auch unter Leitlinien-gerechter Therapie in Spezialambulanzen und Intensivprogrammen erreichen manche PatientInnen mit Anorexia Nervosa kein zufriedenstellendes Ausmaß an seelischer Gesundheit oder Lebensqualität. Kann der palliativmedizinische Ansatz sinnvoll und nutzbringend auf solche Fälle angewendet werden?

\section{Fallbeispiel}

Frau Schulz ${ }^{1}$ litt seit ihrem 16. Lebensjahr an Anorexie mit primärer Amenorrhö, Zwangsstörung und PTBS. Während der ersten stationären Behandlung im 17. Lebensjahr konnte sie ihr Gewicht von 35 auf $54 \mathrm{~kg}$ steigern, nahm nach Entlassung aber rasch bis unter das Aufnahmegewicht ab. Dieser umgekehrte Jo-Jo-Effekt eskalierte in den folgenden Jahren immer weiter: In langen stationären Aufenthalten in renommierten Kliniken mit unterschiedlichen psychotherapeutischen Ausrichtungen nahm Frau Schulz langsam an Gewicht zu, allerdings ohne jemals Normalgewicht zu erreichen. Zwischen den Aufenthalten nahm sie dann trotz ambulanter Fortführung der Psychotherapie rasch bis unter das letzte Aufnahmegewicht ab, wurde im Rahmen eines internistischen Notfalls wieder stationär aufgenommen und nach Stabilisierung in ein psychotherapeutisches Setting verlegt.

Im 22. Lebensjahr erlitt die Patientin einen generalisierten Anfall, vermutlich Hypoglykämie-bedingt, und im anschließenden Intensivstation-Aufenthalt ein Refeeding-Syndrom ${ }^{2}$. Wenige Wochen später wurde sie mit einem BMI von $10,7 \mathrm{~kg} / \mathrm{m}^{2}$ in ein stationäres Essstörungs-Programm aufgenommen. Psychopathologisch fand sich eine ausgeprägte kognitive Inflexibilität: Frau Schulz konnte sich kaum vorstellen, anders zu handeln als sie aktuell handelte oder ein anderes Leben zu führen als das, das sie aktuell führte. Trotz intellektueller Einsicht in die Lebensbedrohlichkeit ihres Untergewichts und der Abwesenheit eines Todeswunsches empfand sie keine Angst vor einem möglichen Versterben (sog. erworbene Furchtlosigkeit [30]). Ihre Motivation zur Teilnahme an einem psychotherapeutischen Programm erschöpfte sich in dem Wunsch, weitere künstliche Ernährung zu verhindern. Dafür versicherte sie wiederholt, zu einer Gewichtszunahme durch Wunschkost entschlossen zu sein. Trotz Einzel-Essbegleitung 3x täglich konnte Frau Schulz ihr gewohntes Verhalten aber nicht ändern, und nahm weiter ab. Als sich auch die Vitalfunktionen verschlechterten (Körpertemperatur bis $33^{\circ} \mathrm{C}$, Blutdruck bis $80 / 50 \mathrm{mmHg}$, BZ bis $20 \mathrm{mg} / \mathrm{dl}$ ) wurde sie auf die Intensivstation zurückverlegt. Trotz der dortigen parenteralen Ernährung verschlechterte sie sich weiter, erlitt zwei generalisierte Anfälle und musste bei ventrikulären Tachyarrhythmien reanimiert werden. Nach einem Monat erfolgte die Rückverlegung in die Klinik für Psychiatrie und Psychotherapie, auch wegen Überforderung des dortigen Teams mit dem krankheitsbedingten Verhalten der Patientin. Frau Schulz hatte zu diesem Zeitpunkt nominell einen BMI von $11,1 \mathrm{~kg} / \mathrm{m}^{2}$, was den Ernährungszustand bei ausgeprägten Beinödemen und pulmonalvenöser Stauung wahrscheinlich überschätzte.

Die Patientin betrachtete die Verlegung auf die Intensivstation als Vertrauensbruch durch das psychiatrische Behandlungsteam (obwohl dies im Vorwege wiederholt als Konsequenz bei ausbleibender Gewichtszunahme dargestellt worden war). Sie war stark belastet von diesem Eingriff in ihre Autonomie und verweigerte jegliche Kooperation, darunter auch jegliche orale Nahrungsaufnahme. Ihr Fragezwang, die kognitiven Einschränkungen im Rahmen des Untergewichts und die geistige Inflexibilität erschwerten die Gesprächsführung und das Treffen von Vereinbarungen. Die Patientin verlor wieder Gewicht, weswegen mit Einverständnis der gesetzlichen Betreuerin eine PEG gelegt wurde.

\footnotetext{
${ }^{1}$ Alle Personen-identifizierenden Daten wurden zum Schutz der Privatsphäre der Patientin und ihrer Angehörigen verfremdet.

${ }^{2}$ Beim schnellem Kostaufbau nach langer Unterernährung kann es zum sog. Refeeding-Syndrom kommen mit Hypomagnesiämie, -kaliämie und -phosphatämie sowie akutem Vitamin B1-Mangel. Mögliche Symptome sind Ödeme, Obstipation, Ataxie, Tremor, generalisierte Krampfanfälle, Hypotension und Herzrhythmusstörungen [14].
} 
Da Frau Schulz ankündigte, diese eigenständig zu entfernen, wurde sie auf die beschützte Station übernommen. Trotz enteraler Zufuhr von 3000 kcal täglich nahm sie auf Grund von Gegenmaßnahmen kaum an Gewicht zu. Beispielsweise nutzte Frau Schulz die Zeit in der Dusche, um im Kopfstand die PEG-Verschlusskappe zu öffnen, wodurch die Nahrung aus dem Magen durch die Sonde in den Abfluss lief. Unter dauerhafter 1:1-Betreuung konnte der BMI schließlich auf $13,4 \mathrm{~kg} / \mathrm{m}^{2}$ gesteigert werden. Diese Gewichtszunahme löste bei der Patientin intensive Ängste und Anspannung aus, sodass sie sich nun auch körperlich gegen jegliche medizinischen Maßnahmen zur Wehr setzte. Da sie dabei sich selbst und die BehandlerInnen gefährdete, wurde die künstliche Ernährung unter Sedierung fortgeführt.

Während dieser insgesamt fünf-monatigen Behandlung konnte somit eine Gewichtssteigerung in einen weniger gefährlichen Bereich erzielt werden. Die therapeutische Beziehung war aber durch die Zwangsmaßnahmen stark belastet, sodass eine Behandlung über die bloße Kalorienzufuhr hinaus nicht mehr möglich war. Auf ihren Wunsch und im Einverständnis mit ihrer gesetzlichen Betreuerin wurde Frau Schulz in eine andere Klinik mit Essstörungs-Schwerpunkt verlegt. Sie verstarb wenige Monate später.

\section{$\underline{\text { Kommentar }}$}

Hier ist es zu einem Teufelskreis gekommen [11, 29]: Mangelnde Mitarbeit seitens der Patientin führt zu somatischer Gefährdung, welche durch Zwangsmaßnahmen für den Moment in Schach gehalten wird. Diese frustrieren aber das Bedürfnis der Patientin nach Autonomie grundlegend, was einerseits Misstrauen gegenüber professionellen Helfern schürt und andererseits dysfunktionale Annahmen über das Selbst verstärkt (z.B. „,verdient Bestrafung“, „böse“) und damit kompensatorisch die Identifizierung mit der Anorexie fördert. Die dementsprechend vermehrten Anorexie-typischen Verhaltensweisen der Patientin führen zu weiterer somatischer Gefährdung, die Behandler sehen sich erneut im Zugzwang, ...

Das Ziel der Zwangsernährung, nämlich eine Besserung der Psychopathologie durch Verbesserung der Energiezufuhr, trat bei Frau Schulz nur in Hinblick auf Vigilanz, Konzentration und Gedächtnisleistung ein. Wesentliche Therapie-erschwerende Symptome wie Emotionsregulationsstörung und Rigidität nahmen hingegen eher zu, vermutlich durch Aktivierung maladaptiver Schemata durch die verschlechterte therapeutische Beziehung und durch das Wieder-Aufleben von Emotionen, die während des Hungerns gedämpft waren [25]. Auch die komorbide Zwangsstörung wird zum Fortbestehen der geistigen Rigidität beigetragen haben.

Die Patientin wurde also von einem hochqualifizierten und engagierten Team in einem störungsspezifischen Setting nach einem leitliniengerechten Konzept behandelt. Trotzdem hat diese Behandlung nicht geholfen und hat sogar zusätzliches Leid geschaffen: Durch die Zwangsmaßnahmen waren die letzten Lebensmonate von Frau Schulz erfüllt von Verzweiflung, Angst, Wut und Ohnmacht.

Es stellt sich daher die Frage, ob Verläufe wie dieser vorhersagbar sind und falls ja, wie solche PatientInnen am besten versorgt werden können.

\section{Severe and Enduring Anorexia Nervosa}

Auch unter Behandlung bleiben etwa $20 \%$ der PatientInnen mit Anorexia Nervosa schwer krank [18]. Basierend auf dem Konzept der Severe and Persistent Mental Illness (SPMI) wurde für Fälle mit mehr als sieben Jahren Krankheitsdauer und besonders schwerer Ausprägung der Begriff Severe and Enduring Anorexia Nervosa (SE-AN) geprägt [37].

Die Mortalität bei SE-AN ist bisher nicht untersucht worden. Da alle Risikofaktoren in dieser Subgruppe gehäuft vor kommen, dürfte die Mortalität bei SE-AN weit über der standardized mortality 
ratio (SMR) von 5 bis 6 liegen, die für Anorexia Nervosa im Allgemeinen berichtet wird [1, 20, 26] ${ }^{3}$. Unterstützung erhält diese These von der deutlich höheren SMR von 7.7 bei zwangsbehandelten (und damit vermutlich schwer kranken) Anorexie-PatientInnen im Vergleich zu freiwillig behandelten $(\mathrm{SMR}=4.6)[38]$.

Um ein Versterben schwerstkranker PatientInnen zu verhindern, werden teilweise medizinische Maßnahmen wie eine künstliche Ernährung unter Zwang angewandt. Dies trifft auf jede fünfte stationäre Aufnahme von Anorexie-PatientInnen zu $[5,6]$. Ethisch legitimiert ist die Anwendung von Zwang hier durch die Annahme, dass mit steigendem Gewicht die Psychopathologie abnehmen und die Patientin ein psychotherapeutisches Angebot annehmen wird, was ihr wiederum die Chance auf ein Leben mit guter Lebensqualität gibt. Und tatsächlich schätzt etwa die Hälfte der von Zwangsmaßnahmen betroffenen Anorexie-PatientInnen diese im Nachhinein als angemessen ein und viele sind dankbar, dass sie nicht ihrer Erkrankung überlassen wurden [32]. Auch führen Behandlungen, die unter Zwang beginnen, im Mittel zu vergleichbaren Gewichtssteigerungen wie freiwillig begonnene Behandlungen $[4,39]$.

\section{Medical Futility in der Psychiatrie}

In manchen Fällen hat Zwangsernährung jedoch keinen Nutzen über eine kurzfristige Gewichtssteigerung hinaus. TherapeutInnen können Anorexie-PatientInnen nicht zwingen, gesund zu werden. Die einzige wirksame Behandlung für die AN, Psychotherapie [42], braucht ein Mindestmaß an freiwilliger, aktiver Kooperation. Fehlt diese, können PatientInnen in einem Teufelskreis aus Zwangsmaßnahmen und somatischer Dekompensation stecken bleiben (s. Fallbeispiel). In einer dänischen Registerstudie von zwangsbehandelten Anorexie-PatientInnen war jede 10. mehr als 100 Mal zwangsbehandelt worden, jede 50. mehr als $500 \mathrm{Mal}$ [6].

Ist das sinnvoll? Wenn 500 Zwangsbehandlungen nicht zu einer dauerhaften Reduktion der Psychopathologie geführt haben, dann wird die 501. Zwangsbehandlung dies höchstwahrscheinlich auch nicht tun. Für ähnliche Situation ist in der angloamerikanischen Intensivmedizin der Begriff medical futility geprägt worden, der sich in etwa mit Aussichtslosigkeit, Sinnlosigkeit und Vergeblichkeit übersetzen lässt [2]. Futility kann zum einen quantitativ definiert werden, also über sehr niedrige Erfolgsaussichten einer Behandlungsoption. Rein statistisch liegt die Wahrscheinlichkeit, nach 500 erfolglosen Zwangsernährungen noch einen Erfolg zu erzielen, zwischen 0,0 und 0,7 \% ${ }^{4}$. Zum anderen kann Futility qualitativ definiert werden als „Behandlung, die zwar physiologisch effektiv ist, aber für den Patienten keinen Nutzen hat" $[2,23]$. Physiologisch effektiv sind fachgerecht durchgeführte Zwangsernährungen - der Ernährungszustand verbessert sich. Aber wenn PatientInnen nach der Zwangsernährung kein psychotherapeutisches Angebot wahrnehmen, dann beschränkt sich der Nutzen der Zwangsernährung auf die bloße Lebensverlängerung.

Lebensverlängernde Maßnahmen ohne Aussicht auf Besserung der Grunderkrankung sind ethisch vertretbar, wenn die Patientin sie wünscht und/oder eine zumindest akzeptable Lebensqualität hat, wie z.B. eine PEG-Ernährung bei Schluckstörungen bei ALS. Bei therapie-refraktärer Anorexie aber wird die lebensverlängernde Maßnahme typischerweise nicht gewünscht, und sie reduziert die Lebensqualität. Die Betroffenen fühlen sich machtlos, bestraft, verängstigt, wie „ein Bürger zweiter Klasse" [29]. Zudem bringt die Zwangsernährung zusätzliche somatische Risiken mit sich, wie Refeeding-Syndrom [14], Infektion, Perforation und Sonden-Dislokation [28], sowie vermehrtes Risikover-

\footnotetext{
${ }^{3}$ Zum Vergleich: Junge Erwachsene, die in der Kindheit eine maligne Erkrankung hatten, haben eine nur geringfügig höhere SMR von 10.8 [24].

${ }^{4}$ Die obere Grenze des 95\%-Konfidenzintervalls der Binomialverteilung nach $n=500$ Versuchen mit $k=0$ Erfolgen liegt bei $p_{o}=0,0074$, somit ergibt sich ein $95 \%-K I$ von $[0,0000 ; 0,0074]$.
} 
halten der PatientInnen zur Anspannungsregulation (z.B. kompletter Verzicht auf Nahrung oder Alkoholexzess nach Entlassung [12]). Übrigens: ob die angestrebte Lebensverlängerung durch Zwangsbehandlungen überhaupt erreicht wird, ist nicht belegt.

\section{Palliativstadium der Anorexia Nervosa}

Wenn Psychotherapie als einzige kurative bzw. krankheitsmodifizierende Behandlung auch mit vorgeschalteten Zwangsmaßnahmen nicht erfolgsversprechend ist, dann muss im einzelnen Fall Unheilbarkeit ${ }^{5}$ der Anorexie angenommen werden. Zusammen mit der eingeschränkten Lebensqualität und der Lebensbedrohlichkeit der Erkrankung (s.o.) ist damit die Definition der WHO für ein Palliativstadium erfüllt [22].

Von dieser allgemeinen Definition müssen störungsspezifische Kriterien abgeleitet werden, um im konkreten Einzelfall entscheiden zu können ob ein Palliativstadium vorliegt. Dieses Adaptationserfordernis erschwert - wie auch bei anderen nicht-onkologischen Erkrankungen wie Herzinsuffizienz und HIV $[15,17]$ - die Implementation einer palliativen Mitbehandlung. Für die Anorexie Nervosa kommen als spezifische Kriterien für ein Palliativstadium alle Risikofaktoren für schlechtes Therapieansprechen und Mortalität in Frage, also

- extrem niedriger BMI (z.B. $\left.<13 \mathrm{~kg} / \mathrm{m}^{2}\right)^{6}$,

- ausgeprägtes Purging-Verhalten,

- psychiatrische Komorbiditäten wie Abhängigkeiten, Zwangs- und Persönlichkeitsstörungen,

- somatische Komorbiditäten wie Diabetes mellitus Typ 1, Niereninsuffizienz,

- lange Krankheitsdauer (z.B. 10 Jahre),

- hohe Anzahl stationärer Aufnahmen und Zwangsmaßnahmen ohne anhaltende Symptomreduktion (z.B. > 3) und

- fehlende Veränderungsbereitschaft, Identifizierung mit/Stolz auf die Erkrankung, Nutzung sog. „pro-Ana“- Internetforen [13, 19, 27, 31, 33, 40].

Konfrontiert mit der Fallvignette einer solchen Patientin hielt eine Stichprobe von Schweizer PsychiaterInnen weitere kurativ ausgerichtete Interventionen für nutzlos und war bereit, eine Reduktion der Lebenserwartung in Kauf zu nehmen, um die Lebensqualität zu verbessern [36].

Skizze einer palliativen Behandlung für Anorexia Nervosa

Kurative und palliative Behandlung schließen sich nicht gegenseitig aus, sondern überlappen und ergänzen sich. Entsprechend muss für jeden Einzelfall neu entschieden werden, wie viele und welche palliativen Elemente zu den kurativ orientierten Behandlungselementen hinzugenommen werden sollen.

Klassisch palliative Elemente sind $\mathrm{u}$. A. ein Fokus auf Lebensqualität und eine partizipative Entscheidungsfindung [21]. PatientInnen werden keinen stark beeinträchtigenden Behandlungen mit minimalen Heilungsaussichten unterzogen (außer sie wünschen es). Im Fall der Anorexie könnte das bedeuten, auf die 50. künstliche Ernährung unter Zwang zu verzichten, wenn die ersten 49 über die bloße Kalorienzufuhr (= Lebensverlängerung) hinaus die Psychopathologie nicht relevant verbessert haben

\footnotetext{
${ }^{5}$ Mit "Unheilbarkeit“ ist hier Nicht-Ansprechen auf professionelle Interventionen gemeint. Spontanremissionen durch Therapie-unabhängige Faktoren (z. B. Auszug aus einem dysfunktionalen Elternhaus) werden ausgeklammert, da sie definitionsgemäß nicht durch professionelles Handeln herstellbar sind.

${ }^{6} \mathrm{BMI}$ als einziges Kriterium - wie im DSM-V mit der Schweregradeinteilung für Anorexie vorgeschlagen - erscheint nicht ausreichend da der BMI mit anderen prognostisch relevanten Faktoren nicht oder nur schwach korreliert $[8,16]$. Hingegen berücksichtigt eine Stadieneinteilung neben dem Querschnitt auch den Längsschnitt-Verlauf einer Erkrankung.
} 
(= Krankheitsmodifikation, s. Tabelle 1). Oder sportliche Aktivität auch bei Frakturgefährdung zu erlauben, um Leid durch Bewegungsdrang zu lindern. Im Zweifelsfall empfiehlt sich die Hinzuziehung einer Palliativmedizinerin und eines Ethikkomittees.

Auch wenn auf Zwangsmaßnahmen zur Lebensverlängerung verzichtet wird, argumentiert auch der palliativ denkende Therapeut für das Leben und damit für eine Gewichtszunahme! Beispielsweise wird er herausarbeiten, dass Ziele der Patientin (z.B. Studieren) wegen der Folgen des Untergewichts (z.B. Konzentrationsstörung) schwerer zu erreichen sind. Gleichzeitig erinnert die Endlichkeit, die im Begriff „palliativ“ mitschwingt, den Therapeuten daran, mit der Patientin und ihren Angehörigen über die eingeschränkte Prognose zu sprechen, ihre Wünsche und Vorstellungen für die letzte Lebensphase zu erfragen und entsprechende Vorbereitungen zu treffen.

Während Art und Weise der Therapiezielfindung also aus der Palliativmedizin entnommen werden, stammen die psychotherapeutischen Interventionen aus der KVT. Insbesondere die Akzeptanz- und Commitment-Therapie bietet eine Fülle von Interventionen zu Werteklärung und Gestaltung eines sinn-erfüllten Lebens [34]. Auch der hohe Strukturierungsgrad der KVT ist sinnvoll, da er den Präferenzen der PatientInnen entgegenkommt und ihre ggf. vorliegenden kognitiven Defizite teilweise kompensieren kann.

Tabelle 1. Elemente einer palliativen Behandlung der Anorexia nervosa

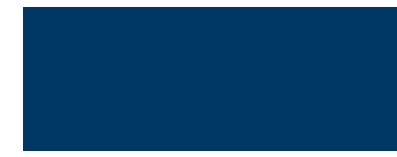

Oberziel

Bestimmung von Unterzielen

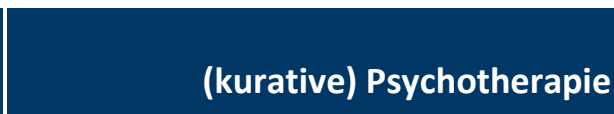

(kurative) Psychotherapie

Reduktion von Problemverhalten (= Krankheitsmodifikation)
Palliativmedizin

\begin{tabular}{|c|c|c|}
\hline Oberziel & $\begin{array}{l}\text { Reduktion von Problemverhalten (= Krank- } \\
\text { heitsmodifikation) }\end{array}$ & Lebensqualität \\
\hline $\begin{array}{l}\text { Bestimmung von } \\
\text { Unterzielen }\end{array}$ & Vorgabe von Verhaltens- und Gewichtszielen & gemeinsames Festlegen individueller Ziele \\
\hline Ansatzpunkt & Verhalten der Patientin & Umstände müssen an Pat. angepasst werden \\
\hline Strukturierungsgrad & $\begin{array}{l}\text { feste Termine, strukturiertes Vorgehen, Ar- } \\
\text { beitsblätter,... }\end{array}$ & flexible Visitenzeiten, freies Gespräch \\
\hline $\begin{array}{l}\text { Auswahl von Inter- } \\
\text { ventionen }\end{array}$ & nach Manual und Kontingent & individuell auf die Pat. zugeschnitten \\
\hline Grenzsetzung & zeitlich kontingentierte Zuwendung & $\begin{array}{l}\text { bedarfsorientierte Zuwendung (innerhalb der } \\
\text { Belastungsgrenzen der Behandlerin) }\end{array}$ \\
\hline Haltung zum Sterben & $\begin{array}{c}=\text { Therapie-Misserfolg } \rightarrow \text { muss durch Zwangs- } \\
\text { maßnahmen verhindert werden }\end{array}$ & $\begin{array}{l}=\text { Teil des Lebens und der Erkrankung } \rightarrow \text { soll } \\
\text { verhindert werden, aber nicht um jeden Preis }\end{array}$ \\
\hline $\begin{array}{l}\text { Priorisierung von } \\
\text { Therapiezielen }\end{array}$ & nach Gefährdung & nach Patientenwunsch \\
\hline
\end{tabular}

Abgrenzung zu ähnlichen Konzepten

PatientInnen im Palliativstadium der Anorexie werden mehrheitlich auch die SE-AN-Kriterien erfüllen. Die Konzepte unterscheiden sich aber in der angenommenen Prognose: Während das SE-AN-Konzept durchaus kurativ bzw. krankheitsmodifizierend ausgerichtet ist, also von der Möglichkeit einer Symp- 
tomreduktion durch professionelle Intervention ausgeht, impliziert das Konzept eines Palliativstadiums deren Aussichtslosigkeit.

Bezüglich der Handlungsempfehlungen ähnelt der hier beschriebene palliative Ansatz in einigen Aspekten dem der KVT-Adaptation für SE-AN [35], dem Community Outreach Partnership Program [41], und dem Recovery Model für Anorexie [9]. Alle diese Ansätze setzen auf Ganzheitlichkeit, stark kollaborative therapeutische Beziehung, Förderung von Hoffnung, Selbstverantwortung und WerteOrientierung. Da Recovery aber vom Patienten selbst ausgehen muss, lässt sich dieser Ansatz kaum anwenden auf PatientInnen ohne Problembewusstsein. Bei stark ich-syntonen Erkrankungen wie der Anorexia nervosa sind ihm also Grenzen gesetzt, ebenso am unmittelbaren Lebensende. Auch die KVT-Adaptation für SE-AN und das Community Outreach Partnership Program geben keine Empfehlungen für PatientInnen, die Behandlungen mit vitaler Indikation ablehnen. Der palliative Ansatz hingegen bietet BehandlerInnen gerade in diesen schwierigen Situationen Orientierung.

\section{Nutzen des Begriffs „palliativ“}

Aber ist das bisher Gesagte nicht einfach gute psychiatrische Behandlung? Sicher. Gute Medizin beinhaltet immer kurative und palliative Aspekte. Ist der Begriff „palliativ“ dann überflüssig? Nein. Wie alle Menschen neigen ÄrztInnen gerade in Stresssituationen dazu, den Handlungsimpulsen ihres aktiven Mindsets zu folgen ${ }^{7}$. Bei ÄrztInnen ist meistens das kurative Mindset aktiv, weswegen sie bei Gefährdung einer Patientin lebensverlängernde Maßnahmen einleiten. Wenn die Krankheit aber nicht auf krankheitsmodifizierende Interventionen anspricht, ein letaler Verlauf hochwahrscheinlich und die Lebensqualität niedrig sind, dann ist ein solcher Automatismus nicht unbedingt im Sinne der Patientin. Entsprechend konnte für die somatische Palliativmedizin gezeigt werden, dass ein Mindset-Wechsel notwendig ist für eine gute Betreuung am Lebensende [10].

Bei PatientInnen mit Anorexie dürfte die Aufgabe des kurativen Mindsets noch schwerer fallen, da sie typischerweise jung sind und vermeintlich leicht gerettet werden könnten („Einfach mehr essen!“). Die Einordnung als palliativ kann hier als stete Erinnerung an die schlechte Prognose und die Aussichtslosigkeit kurativer/krankheitsmodifizierender Interventionen dienen. Geistige, personelle und finanzielle Ressourcen, welche im kurativen Mindset für solche Interventionen verwandt worden wären, können dann zur Verbesserung der Lebensqualität eingesetzt werden: Wie können wir ihr ermöglichen, regelmäßig ihr Pferd zu besuchen? Er möchte sich mit seiner Tante aussöhnen, welche Unterstützung braucht er dafür von uns [3]?

Neben der Strukturierung des eigenen Denkens kann der Begriff „palliativ“ helfen, Behandlungsziele und -prioritäten rasch zwischen BehandlerInnen auszutauschen. Er kann zu verstärkter Vernetzung und Kooperation von Psychiatrie, Palliativpsychologie und Palliativmedizin beitragen und die gegenseitige Rezeption von Ansätzen und Forschungsergebnissen erleichtern.

Wichtige Forschungsfragen sind die Weiterentwicklung von Stadieneinteilungen der Anorexia nervosa sowie die Bestimmung von Inzidenz, Prävalenz und Mortalität der einzelnen Stadien, die Adaptation des Konzepts der medizinischen Nutzlosigkeit für die Psychiatrie, die empirische Überprüfung der hier vorgeschlagenen Kriterien für das Palliativstadium der Anorexia Nervosa und die Erarbeitung von Empfehlungen für die Betreuung von Anorexie-PatientInnen am unmittelbaren Lebensende.

Fazit für die Praxis

\footnotetext{
${ }^{7}$ Relativ stabiles mentales Netz von Einstellungen, Erinnerungen, Werten, Gefühlen und automatischen Verhaltensweisen, das mitbestimmt, wie wir eine Situation interpretieren und wie wir auf sie reagieren [7].
} 
- In seltenen Fällen verläuft eine Anorexia nervosa so schwer, dass sie keiner krankheitsmodifizierend ausgerichteten Behandlung zugänglich und mit einer hohen Mortalität assoziiert ist. Die betroffenen PatientInnen befinden sich in einem Palliativstadium.

- Bei schweren und therapie-refraktären Verlaufsformen sollte bei klinischen Entscheidungen, insbesondere bei der Erwägung von Zwangsmaßnahmen, neben der Lebensverlängerung auch die subjektive Lebensqualität der Patientin berücksichtigt werden. Ebenso sollte geschätzt werden, wie wahrscheinlich die Erreichung des angestrebten Behandlungsziels mit der erwogenen (Zwangs-)Maßnahme ist.

- Im Zweifelsfall empfiehlt sich die Hinzuziehung einer Palliativmedizinerin und des klinischen Ethikkomittees.

Literatur

1. Arcelus J, Mitchell AJ, Wales J et al. (2011) Mortality rates in patients with anorexia nervosa and other eating disorders. A meta-analysis of 36 studies. Arch. Gen. Psychiatry 68:724-731

2. Becker G, Blum H (2004) "Medical Futility”: Der Arzt im Spannungsfeld von Behandlungsauftrag und Behandlungsbegrenzung. DMW-Deutsche Medizinische Wochenschrift 129:1694-1697

3. Berk M, Berk L, Udina M et al. (2012) Palliative models of care for later stages of mental disorder: maximizing recovery, maintaining hope, and building morale. Aust. N. Z. J. Psychiatry 46:92-99

4. Born C, De La Fontaine L, Winter B et al. (2015) First results of a refeeding program in a psychiatric intensive care unit for patients with extreme anorexia nervosa. BMC Psychiatry 15:57

5. Clausen L, Jones A (2014) A systematic review of the frequency, duration, type and effect of involuntary treatment for people with anorexia nervosa, and an analysis of patient characteristics. Journal of eating disorders 2:29

6. Clausen L, Larsen JT, Bulik CM et al. (2018) A Danish register-based study on involuntary treatment in anorexia nervosa. Int. J. Eat. Disord. 51:1213-1222

7. Crum AJ, Salovey P, Achor S (2013) Rethinking stress: The role of mindsets in determining the stress response. J. Pers. Soc. Psychol. 104:716

8. Dakanalis A, Alix Timko C, Colmegna F et al. (2018) Evaluation of the DSM-5 severity ratings for anorexia nervosa in a clinical sample. Psychiatry Res. 262:124-128

9. Dawson L, Rhodes P, Touyz S (2014) The recovery model and anorexia nervosa. Aust. N. Z. J. Psychiatry 48:1009-1016

10. De Korte-Verhoef MC, Pasman HRW, Schweitzer BP et al. (2015) How could hospitalisations at the end of life have been avoided? A qualitative retrospective study of the perspectives of general practitioners, nurses and family carers. PloS one 10:e0118971

11. Douzenis A, Michopoulos I (2015) Involuntary admission: the case of anorexia nervosa. Int. J. Law Psychiatry 39:31-35

12. Dyer C (2012) Anorexic woman cannot be force fed, judge rules. Br. Med. J. 345

13. Franko DL, Keshaviah A, Eddy KT et al. (2013) A longitudinal investigation of mortality in anorexia nervosa and bulimia nervosa. A. J. Psychiatry 170:917-925

14. Friedli N, Stanga Z, Sobotka L et al. (2017) Revisiting the refeeding syndrome: results of a systematic review. Nutrition 35:151-160

15. Gelfman LP, Kavalieratos D, Teuteberg WG et al. (2017) Primary palliative care for heart failure: what is it? How do we implement it? Heart Fail Rev 22:611-620

16. Gianini L, Roberto CA, Attia E et al. (2017) Mild, moderate, meaningful? Examining the psychological and functioning correlates of DSM-5 eating disorder severity specifiers. Int. J. Eat. Disord. 50:906-916

17. Harding R (2018) Palliative care as an essential component of the HIV care continuum. The Lancet HIV 5:e524-e530 
18. Keel PK, Brown TA (2010) Update on course and outcome in eating disorders. Int. J. Eat. Disord. 43:195-204

19. Keel PK, Dorer DJ, Eddy KT et al. (2003) Predictors of mortality in eating disorders. Arch. Gen. Psychiatry 60:179-183

20. Keshaviah A, Edkins K, Hastings ER et al. (2014) Re-examining premature mortality in anorexia nervosa: A meta-analysis redux. Compr. Psychiatry 55:1773-1784

21. Leitlinienprogramm Onkologie (2015) Palliativmedizin für Patienten mit einer nicht heilbaren Krebserkrankung. In: AWMF-Registernummer: 128/0010L.

22. Lindblad A, Helgesson G, Sjöstrand M (2019) Towards a palliative care approach in psychiatry: do we need a new definition? J. Med. Ethics 45:26-30

23. Lopez A, Yager J, Feinstein RE (2010) Medical futility and psychiatry: palliative care and hospice care as a last resort in the treatment of refractory anorexia nervosa. Int. J. Eat. Disord. 43:372-377

24. Mertens AC, Yasui Y, Neglia JP et al. (2001) Late Mortality Experience in Five-Year Survivors of Childhood and Adolescent Cancer: The Childhood Cancer Survivor Study. J. Clin. Oncol. 19:3163-3172

25. Munro C, Randell L, Lawrie SM (2017) An Integrative Bio-Psycho-Social Theory of Anorexia Nervosa. Clinical Psychology \& Psychotherapy 24:1-21

26. Papadopoulos FC, Ekbom A, Brandt L et al. (2009) Excess mortality, causes of death and prognostic factors in anorexia nervosa. The British Journal of Psychiatry 194:10-17

27. Rouleau CR, Von Ranson KM (2011) Potential risks of pro-eating disorder websites. Clin. Psychol. Rev. 31:525-531

28. Schrag SP, Sharma R, Jaik NP et al. (2007) Complications related to percutaneous endoscopic gastrostomy (PEG) tubes. A comprehensive clinical review. Journal of Gastrointestinal and Liver Diseases 16:407

29. Seed T, Fox J, Berry K (2016) Experiences of Detention under the Mental Health Act for Adults with Anorexia Nervosa. Clin Psychol Psychother 23:352-362

30. Selby EA, Smith AR, Bulik CM et al. (2010) Habitual starvation and provocative behaviors: two potential routes to extreme suicidal behavior in anorexia nervosa. Behav. Res. Ther. 48:634645

31. Steinhausen HC (2002) The outcome of anorexia nervosa in the 20th century. A. J. Psychiatry 159:1284-1293

32. Tan JO, Stewart A, Fitzpatrick R et al. (2010) Attitudes of patients with anorexia nervosa to compulsory treatment and coercion. Int. J. Law Psychiatry 33:13-19

33. Tierney S, Fox JR (2009) Chronic anorexia nervosa: A Delphi study to explore practitioners' views. Int. J. Eat. Disord. 42:62-67

34. Timko CA, Eifert G, Harres A (2013) Akzeptanz-und Commitmenttherapie bei Anorexia nervosa: Ein Leitfaden für die Behandlung mit ACT. Mit Online-Materialien. Beltz

35. Touyz S, Le Grange D, Lacey $\mathrm{H}$ et al. (2013) Treating severe and enduring anorexia nervosa: a randomized controlled trial. Psychol. Med. 43:2501-2511

36. Trachsel M, Hodel MA, Irwin SA et al. (2019) Acceptability of palliative care approaches for patients with severe and persistent mental illness: a survey of psychiatrists in Switzerland. BMC Psychiatry 19:111

37. Treasure J, Stein D, Maguire S (2015) Has the time come for a staging model to map the course of eating disorders from high risk to severe enduring illness? An examination of the evidence. Early intervention in psychiatry 9:173-184

38. Ward A, Ramsay R, Russell G et al. (2015) Follow-up mortality study of compulsorily treated patients with anorexia nervosa. Int. J. Eat. Disord. 48:860-865

39. Watson TL, Bowers WA, Andersen AE (2000) Involuntary treatment of eating disorders. A. J. Psychiatry 157:1806-1810 
40. Westmoreland P, Mehler PS (2016) Caring for Patients With Severe and Enduring Eating Disorders (SEED): Certification, Harm Reduction, Palliative Care, and the Question of Futility. J Psychiatr Pract 22:313-320

41. Williams KD, Dobney T, Geller J (2010) Setting the eating disorder aside: An alternative model of care. European Eating Disorders Review: The Professional Journal of the Eating Disorders Association 18:90-96

42. Zipfel S, Giel KE, Bulik CM et al. (2015) Anorexia nervosa: aetiology, assessment, and treatment. Lancet Psychiatry 2:1099-1111

Einhaltung ethischer Richtlinien

Interessenkonflikt. A. Westermair, K. Perrar und U. Schweiger geben an, dass kein Interessenkonflikt besteht.

Dieser Beitrag beinhaltet keine Studien an Menschen oder Tieren.

Danksagungen

Die Autoren bedanken sich herzlich bei den anderen Mitgliedern der joint taskforce der DGPPN, der DGGPP und der DGP für die konstruktive Diskussion der Inhalte dieses Artikels. ALW bedankt sich zusätzlich herzlich bei Dr. Norman Kripke für die immer geduldige und hilfreiche oberärztliche Betreuung auf der Palliativstation und bei Prof. Fritz Hohagen für die konstruktive Kritik des Manuskripts. 